\section{SAT0236 SAFETY AND DISEASE ACTIVITY CHANGES IN AN EXTENSION OF A PHASE IIB STUDY OF ATACICEPT IN PATIENTS WITH SLE (ADDRESS II)}

D.J. Wallace ${ }^{1}$, D. Isenberg ${ }^{2}$, S. Wax ${ }^{3}$, A. $\mathrm{Kao}^{3}$, P. Chang ${ }^{3}$, P.A. Fraser ${ }^{3}$, J.T. Merrill ${ }^{4} .{ }^{1}$ Cedars-Sinai Medical Center, University of California Los Angeles, Los Angeles, United States; ${ }^{2}$ University College London, London, United Kingdom; ${ }^{3} E M D$ Serono Research \& Development Institute, Inc. (a business of Merck KGaA, Darmstadt, Germany), Billerica; ${ }^{4}$ Oklahoma Medical Research Foundation, Oklahoma City, United States

Background: We previously reported results of the 24-week phase IIb ADDRESS II study of atacicept, which demonstrated clinical response in patients with autoantibody-positive SLE, particularly those with high disease activity (HDA; SLEDAI $2 \mathrm{~K} \geq 10$ at screening).

Objectives: This extension study of ADDRESS II evaluated safety and disease activity in patients with SLE given continued atacicept treatment to week 48 (NCT01972568).

Methods: Atacicept was given as weekly subcutaneous injection to completers of ADDRESS II on average for an additional $\geq 24$ weeks (i.e. $\geq 48$ weeks total from Day 1 of ADDRESS II). Patients already receiving atacicept continued on the same dose (75 $\mathrm{mg}$ or $150 \mathrm{mg}$ ); those receiving placebo (PBO) switched to atacicept $150 \mathrm{mg}$ (PBO/150 mg).

Results: Of 262 patients completing ADDRESS II, 253 (95\%) entered the extension ( $\mathrm{PBO} / 150 \mathrm{mg} \mathrm{n}=83 ; 75 \mathrm{mg} \mathrm{n}=82 ; 150 \mathrm{mg} \mathrm{n}=88$ ). Demographics were balanced between groups, and most patients were female (91.3\%) and white $(70 \%)$. All three treatment groups had similar rates of treatment-emergent adverse events (TEAEs), TEAEs leading to treatment discontinuation, serious TEAEs, and serious/severe infection (Table 1). Two deaths occurred in the $150 \mathrm{mg}$ arm, considered unrelated to treatment (reported events of stroke and abdominal pain with hematemesis). In the ITT population ( $n=253$ ), SLE responder index (SRI)-4 rates were maintained between weeks 24 and 48 in the original atacicept groups ( $75 \mathrm{mg} 56.9$ vs $55.9 \% ; 150 \mathrm{mg} 53.8$ vs $56.7 \%$ ) and moderately increased in the PBO/150 mg group (44.0 vs $48.0 \%$ ). This improvement was greater for SRI-6 in the HDA subpopulation (PBO/150 mg $28.8 \%$ at week 24 vs $42.3 \%$ at week $48 ; 75 \mathrm{mg} 41.8$ vs $54.5 \% ; 150 \mathrm{mg} 54.9$ vs $60.8 \%$; Figure 1). The proportion of HDA patients achieving SLEDAI scores $\leq 2$ was also maintained in the atacicept groups (75 mg 20.0 vs $21.8 \% ; 150 \mathrm{mg} 37.3$ vs $39.2 \%$ ) and increased in the PBO/150 mg group (13.5 vs $19.2 \%)$. In the ITT and HDA populations, most flares occurred before week 24, with reduced risk of severe (BILAG A) and moderate/severe (BILAG A/2B) flares afterwards. Some increase in complement C3 and C4, and decrease in anti-dsDNA antibodies occurred after week 24. Serum IgG decreased moderately, without severe hypogammaglobulinemia (IgG $<3 \mathrm{~g} / \mathrm{L}$ ) in any patients.

Table 1. Summary of adverse events

\begin{tabular}{lccc}
\hline Adverse events, $\mathrm{n}(\%)$ & $\begin{array}{c}\mathrm{PBO} / 150 \mathrm{mg}^{*} \\
(\mathrm{n}=83)\end{array}$ & $\begin{array}{c}\text { Atacicept 75 mg } \\
(\mathrm{n}=82)\end{array}$ & $\begin{array}{c}\text { Atacicept 150 mg } \\
(\mathrm{n}=88)\end{array}$ \\
\hline Any TEAEs & $62(74.7)$ & $63(76.8)$ & $65(73.9)$ \\
TEAEs leading to treatment discontinuation & $6(7.0)$ & $4(4.9)$ & $5(5.7)$ \\
Infections and infestations & $27(32.5)$ & $35(42.7)$ & $43(48.9)$ \\
Herpes zoster & $1(1.2)$ & 0 & $1(1.2)$ \\
Influenza & $1(1.2)$ & $1(1.2)$ & $2(2.3)$ \\
Serious TEAEs & $15(18.1)$ & $10(12.2)$ & $10(11.4)$ \\
Serious/severe infections & $4(4.8)$ & $4(4.9)$ & $5(5.7)$ \\
Death & 0 & 0 & $2(2.3 \%)^{\dagger}$ \\
\hline
\end{tabular}

PBO, placebo; TEAE, treatment-emergent adverse event. ${ }^{*}$ Patients receiving PBO in ADDRESS II who switched to atacicept $150 \mathrm{mg}$. ${ }^{\dagger}$ Due to 1) stroke and 2) hematemesis.

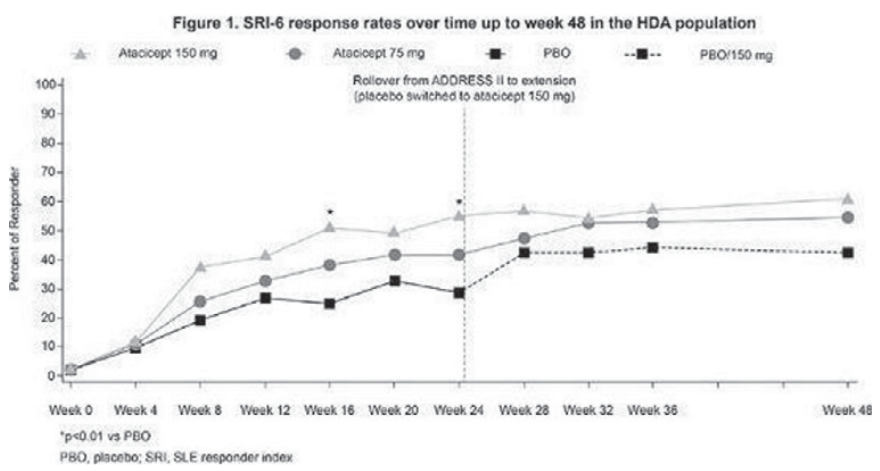

Conclusions: There were no new safety signals with atacicept between week 24 and 48, and clinical responses achieved during the first 24 weeks were maintained.

Acknowledgements: The study was sponsored by EMD Serono Research \& Development Institute Inc., USA (a business of Merck KGaA, Germany). Medical writing support was provided by Bioscript Science, UK, and funded by Merck KGaA, Germany.

Disclosure of Interest: D. Wallace Consultant for: EMD Serono Research \& Development Institute, Inc. (a business of Merck KGaA, Darmstadt, Germany), Billerica, MA, USA, D. Isenberg Consultant for: EMD Serono Research \&
Development Institute, Inc. (a business of Merck KGaA, Darmstadt, Germany), Billerica, MA, USA, S. Wax Employee of: EMD Serono Research \& Development Institute, Inc. (a business of Merck KGaA, Darmstadt, Germany), Billerica, MA, USA, A. Kao Employee of: EMD Serono Research \& Development Institute, Inc. (a business of Merck KGaA, Darmstadt, Germany), Billerica, MA, USA, P. Chang Employee of: EMD Serono Research \& Development Institute, Inc. (a business of Merck KGaA, Darmstadt, Germany), Billerica, MA, USA, P. Fraser Employee of: EMD Serono Research \& Development Institute, Inc. (a business of Merck KGaA, Darmstadt, Germany), Billerica, MA, USA, J. Merrill Consultant for: Anthera Pharmaceuticals, Lilly, EMD Serono, GlaxoSmithKline and Biogen DOI: 10.1136/annrheumdis-2017-eular.3665

\section{SAT0237 BONE MARROW AS A TARGET ORGAN OF SYSTEMIC LUPUS ERYTHEMATOSUS: ANALYSIS OF CASES WITH AUTOIMMUNE MYELOFIBROSIS}

D. Üsküdar Cansu ${ }^{1}$, H. Üsküdar Teke ${ }^{2}$, S. Işıksoy ${ }^{3}$, C. Korkmaz ${ }^{1} .{ }^{1}$ Eskişehir Osmangazi University, School of Medicine, Rheumatology; ${ }^{2}$ Eskişehir

Osmangazi University, School of Medicine, Hematology; ${ }^{3}$ Eskişehir Osmangazi University, School of Medicine, Pathology, Eskişehir, Turkey

Background: Cytopenia in the course of systemic lupus erythematosus (SLE) may be due to multiple factors. One of these factors can be SLE-associated autoimmune myelofibrosis (AIMF). However, the frequency of SLE-associated AIMF is not well known and the role of clinical and laboratory parameters in the development of AIMF is not clear.

Objectives: Our aim was to identify the frequency of SLE-associated AIMF and compared SLE-associated AIMF group with non-AIMF group in terms of clinical findings and morphological properties of the bone marrow (BM) in cytopenic SLE patients.

Methods: We retrospectively analyzed 224 SLE patients' files who met 1997 revised Classification criteria for SLE. BM aspirates and trephine biopsies were re-examinded. Patients were divided into two groups according to whether they had myelofibrosis or not (AIMF and non-AIMF groups). Concurrent SLE organ involvements, and drugs given pre- and post-AIMF were recorded. BM cellularity, the presence of fibrosis (reticulin or collagen) and grade of fibrosis, the presence of dysplasia, and lymphoid infiltration were recorded.

Results: $45(20 \%)$ of 224 SLE patients were found to experienced BM biopsy due to cytopenia. Four patients were excluded from analyses (2 druginduced cytopenia, 1 lymphoma, 1 insufficient BM biopsy samples). While AIMF was detected in $29(70.7 \%)$ of the 41 patients, 12 patients did not have AIMF. All patients with AIMF had reticulin fibrosis, 2 patients $(6.9 \%)$ had also collagen fibrosis. Between the AIMF and non-AIMF groups, no differences were identified in terms of erythrocyte sedimentation rate (ESR), C-reactive protein (CRP), SLEDAI, BM cellularity, or BM dysplastic changes ( $p=0.989, p=0.387$, $p=0.788, p=0.672$, and $p=0.494$, respectively). In the AIMF group, 27 patients responded to immunosuppressive therapy and corticosteroids, but 2 patients were unresponsive. The response time was longer for the AIMF group compared to the non-AIMF group $(3.3 \pm 3.1$ months vs $1.7 \pm 1.2$ months, $p=0.091)$. Correlation analysis revealed that higher the grade of $\mathrm{BM}$ fibrosis, longer the response time $(r=0.471, p=0.002)$.

Conclusions: AIMF may be underestimated in SLE patients. AIMF as an additional factor for cytopenia in SLE patients may lead to delayed response to appropriate therapy, which was dependent on the presence of high grade fibrosis. Acknowledgements: None declared

Disclosure of Interest: None declared

DOI: 10.1136/annrheumdis-2017-eular.4330

\section{SAT0238 GLUCOCORTICOID WITHDRAWAL IN AN INCEPTION COHORT OF LUPUS NEPHRITIS PATIENTS}

S. Wautier, F. Tamirou, S. Nieuwland-Husson, F.A. Houssiau. Rheumatology Department, Cliniques Universitaires Saint-Luc, Bruxelles, Belgium

Background: Few studies have addressed glucocorticoid (GC) withdrawal in lupus nephritis (LN). Yet, this remains a pivotal issue due to GC-induced side effects on the one hand, and to the risk of relapse on the other hand.

Objectives: We reviewed the data of the Louvain Lupus Nephritis Inception Cohort (LOULUNIC) i): to determine the percentage of patients able to permanently or transiently stop GC; ii): to compare their baseline and follow-up characteristics to patients who never stopped; and iii): to assess the consequences of GC withdrawal.

Methods: Ninety patients with new-onset biopsy-proven LN were included. All were under the care of the same senior physician $(F A H)$ during follow-up. Clinical, pathological and biological data were extracted from our data base. The SLICC/ACR-DI was assessed at last visit. Unpaired t-tests, Mann-Whitney tests and ANOVA were used, as appropriate.

Results: Out of 90 patients with incident LN, 43 (48\%) ever stopped GC (group E), of which 32 permanently (group P). Median time to stop GC was 37 months. 47 patients $(52 \%)$ never stopped GC (group N).

At baseline, serum creatinine, UP/C ratio, ISN/RPS classes, activity and chronicity indices did not differ between groups, nor did the mean initial dose of methylprednisolone (MP) (N: $28 \mathrm{mg} / \mathrm{d}$; E: $32 \mathrm{mg} / \mathrm{d} ; \mathrm{P}: 31 \mathrm{mg} / \mathrm{d}$ ), the use of IV MP pulses (82 